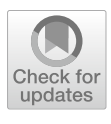

Cite as

Nano-Micro Lett.

(2020) 12:153

Received: 27 April 2020

Accepted: 15 June 2020

Published online: 18 July 2020

(C) The Author(s) 2020

\section{Solvent-Free Synthesis of Ultrafine Tungsten Carbide Nanoparticles-Decorated Carbon Nanosheets for Microwave Absorption}

\author{
Yunlong Lian ${ }^{1}$, Binhua Han ${ }^{1}$, Dawei Liu ${ }^{1}$, Yahui Wang ${ }^{1}$, Honghong Zhao ${ }^{1}$, Ping Xu ${ }^{1}$, \\ Xijiang $\operatorname{Han}^{1 凶}$, Yunchen $\mathrm{Du}^{1} \bowtie$ \\ Yunlong Lian and Binhua Han contributed equally to this work. \\ $\triangle$ Xijiang Han, hanxijiang@hit.edu.cn; Yunchen Du, yunchendu@ hit.edu.cn \\ 1 MIIT Key Laboratory of Critical Materials Technology for New Energy Conversion and Storage, \\ School of Chemistry and Chemical Engineering, Harbin Institute of Technology, Harbin 150001, \\ People's Republic of China
}

\title{
HIGHLIGHTS
}

- Ultrafine tungsten carbide nanoparticles-decorated carbon nanosheets were successfully fabricated via a simple solvent-free strategy.

- The chemical composition of tungsten carbide/carbon composites can be easily manipulated by the weight ratio of dicyandiamide to ammonium metatungstate.

- The advantages in good performance and simple preparation provide a promising prospect for the application of these tungsten carbide/ carbon composites.

ABSTRACT Carbides/carbon composites are emerging as a new kind of binary dielectric systems with good microwave absorption performance. Herein, we obtain a series of tungsten carbide/carbon composites through a simple solvent-free strategy, where the solid mixture of dicyandiamide (DCA) and ammonium metatungstate (AM) is employed as the precursor. Ultrafine cubic $\mathrm{WC}_{1-x}$ nanoparticles (3-4 nm) are in situ generated and uniformly dispersed on carbon nanosheets. This configuration overcomes some disadvantages of conventional carbides/carbon composites and is greatly helpful for electromagnetic dissipation. It is found that the weight ratio of DCA to AM can regulate chemical composition of these composites, while less impact on the average size of $\mathrm{WC}_{1-x}$ nanoparticles. With

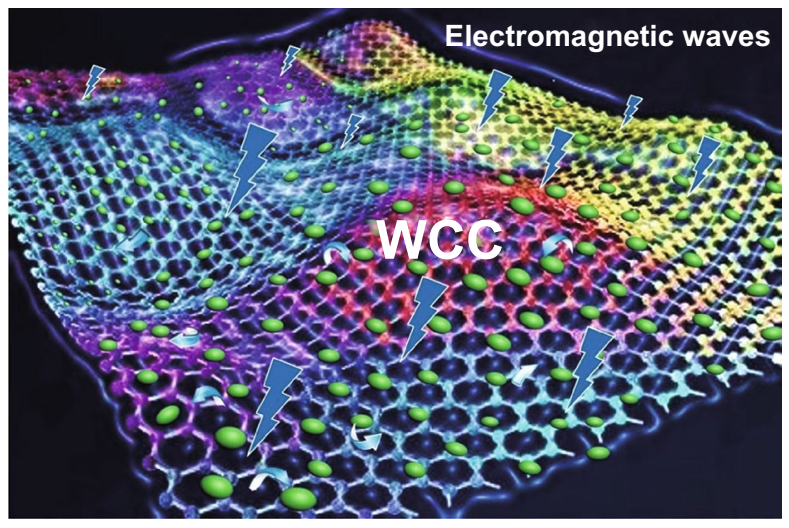
the increase in carbon nanosheets, the relative complex permittivity and dielectric loss ability are constantly enhanced through conductive loss and polarization relaxation. The different dielectric properties endow these composites with distinguishable attenuation ability and impedance matching. When DCA/AM weight ratio is 6.0, the optimized composite can produce good microwave absorption performance, whose strongest reflection loss intensity reaches up to - 55.6 $\mathrm{dB}$ at 17.5 GHz and qualified absorption bandwidth covers $3.6-18.0 \mathrm{GHz}$ by manipulating the thickness from 1.0 to $5.0 \mathrm{~mm}$. Such a performance is superior to many conventional carbides/carbon composites.

KEYWORDS Solvent-free synthesis; Tungsten carbide/carbon composite; Ultrafine nanoparticle; Microwave absorption; Dielectric loss 


\section{Introduction}

Electromagnetic (EM) pollution has become a global concern owing to its potential threats in physical health, equipment operation, and information security [1-4]. Although the shielding strategy can produce powerful effectiveness in individual protection, the reflection principle therein makes it invalid to alleviate those side effects of EM technology [5, 6]. In the past two decades, microwave absorption emerged as an advanced and sustainable alternative for the precaution of EM pollution, because this promising strategy was established on the fundamental energy conversion through the interaction between EM waves and microwave-absorbing materials (MAMs) [7, 8]. Among various functional materials with unique EM characteristics, carbon materials are receiving more and more attention due to their desirable advantages in tunable dielectric property, low density, chemical inertness, and designable microstructure [9-11]. It is unfortunate that single-component carbonaceous MAMs easily suffer from mismatched impedance with that of free space, which will result in their poor microwave absorption performance as most of EM waves are reflected off at the interface rather than being transmitted into MAMs [3, 12]. In an effort to reinforce the consumption of EM energy, there are numerous efforts that have been devoted to carbon-based composites by introducing some magnetic components (i.e., magnetic metals and ferrites) [13-17], while the intrinsic drawbacks of magnetic components in carbon matrix, including easy corrosion, weak magnetic loss, and Curie temperature limitation, restrain the practical application of these composites to some extent [18-20]. As a result, many researchers started to focus on the fabrication of nonmagnetic systems that combine carbon materials and secondary dielectric components [21-23]. Our group recently designed core-shell $\mathrm{BaTiO}_{3} @ \mathrm{C}$ microspheres as a binary dielectric system, and we found that it displayed comparable microwave absorption performance but superior corrosion resistance to those common magnetic carbon-based composites [24].

Carbides, as a kind of special ceramics, have good mechanical, physical, and chemical properties that make them active in many fields ranging from energy storage to catalysis $[25,26]$. In particular, their intrinsic characteristics of polarization relaxation also render them as popular dielectric components to couple with carbon materials for
EM attenuation [27, 28]. A lot of successful examples have demonstrated the positive synergistic effects between carbon and carbides that could enhance the microwave absorption performance of these composites effectively [29-31]. However, in most cases, the selection of carbides was usually limited to $\mathrm{SiC}$ or $\mathrm{Ti}_{3} \mathrm{C}_{2}$ Mxenes. It is well known that either $\mathrm{SiC}$ or $\mathrm{Ti}_{3} \mathrm{C}_{2}$ has very large particle size even up to several microns, which means that these carbides particles cannot fully contact with carbon matrix, and thus, the contribution from interfacial polarization may be insufficient in such carbon/carbide composites [29, 32]. More recently, some attempts were made to decorate carbon matrix with some unconventional carbide particles, whose ultrafine particle size could generate good dispersion and abundant interfaces, resulting in a significant enhancement in microwave absorption [33-37]. For example, Dai et al. pioneered the involvement of $\mathrm{Mo}_{2} \mathrm{C}$ nanoparticles in carbon matrix through the pyrolysis of $\mathrm{Cu}-\mathrm{Mo}$-based metal organic frameworks (MOFs) and the subsequent removal of $\mathrm{Cu}$ species, and they confirmed that the synergistic effect of carbon frameworks and $\mathrm{Mo}_{2} \mathrm{C}$ nanoparticles were responsible for the good reflection loss (RL) characteristics [34]. Li et al. conducted the coating of carbonaceous layer on pre-prepared $\mathrm{MoO}_{3}$ nanowires through hydrothermal carbonization of glucose solution and then transformed this precursor into final $\mathrm{Mo}_{2} \mathrm{C} @ \mathrm{C}$ nanowires under a high-temperature inert atmosphere, and they demonstrated that the composition and microstructure were favorable for the consumption of EM energy by multiple polarization relaxations [35]. In our previous work, we also harvested $\mathrm{Mo}_{2} \mathrm{C} / \mathrm{C}$ composites from Mo-substituted ZIF-8, whose microwave absorption performance was found to be superior to those counterparts with $\mathrm{SiC}$ and $\mathrm{Ti}_{3} \mathrm{C}_{2}$ [37]. Although some positive achievements have been identified in these carbon-based composites with ultrafine carbide particles, it is worth noting that the formation of such composites is always complex and time-consuming. Therefore, a simple method is highly desirable to produce carbon-based composites with uniform dispersion of ultrafine carbide particles.

Herein, we report a solvent-free route for tungsten carbide/carbon composites, which only requires grinding the mixture of dicyandiamide (DCA, carbon source) and ammonium metatungstate (AM, tungstate source) before hightemperature pyrolysis. The final products are composed of highly dispersed ultrafine tungsten carbide nanoparticles (ca. 3-4 nm) and amorphous carbon matrix, and their EM 
properties and RL characteristics can be easily regulated by the weight ratio of DCA to AM. When the composition is optimized, the resultant tungsten carbide/carbon can produce good microwave absorption with the strongest RL intensity of $-55.6 \mathrm{~dB}$ and the effective absorption bandwidth of $14.4 \mathrm{GHz}(3.6-18.0 \mathrm{GHz}, 1.0-5.0 \mathrm{~mm})$. Compared with those common wet chemical ways, this solvent-free strategy is obviously advantageous of simple operation, saving time, environmental benign, and scale-up production [38, 39], and thus, this study may open a new avenue for the fabrication of carbide/carbon composites.

\section{Experimental Section}

\subsection{Synthesis}

A typical procedure for tungsten carbide/carbon composite is illustrated in Fig. 1. First, $0.5 \mathrm{~g}$ of AM was added into an agate mortar, and then, a required amount of DCA was also introduced. The mixture was sufficiently ground for $15 \mathrm{~min}$. After that, the mixture was transferred into a porcelain boat and pyrolyzed in a horizontally tubular furnace under $\mathrm{N}_{2}$ nitrogen at $400{ }^{\circ} \mathrm{C}$ for $0.5 \mathrm{~h}$ and $800{ }^{\circ} \mathrm{C}$ for $5 \mathrm{~h}$, respectively. The heating rates from room temperature to $400{ }^{\circ} \mathrm{C}$ and from 400 to $800^{\circ} \mathrm{C}$ were 2 and $5{ }^{\circ} \mathrm{C} \mathrm{min}{ }^{-1}$, respectively. The final products were denoted as WCC- $r$, where $r$ referred to the weight ratio of DCA to AM.

\subsection{Characterization}

Powder X-ray diffraction (XRD) data were recorded on a Rigaku D/MAXRC X-ray diffractometer (45.0 kV, 50.0 mA) using $\mathrm{Cu} \mathrm{K} \alpha$ source. Raman spectra were recorded on a confocal Raman spectroscopic system (Renishaw, In Via)

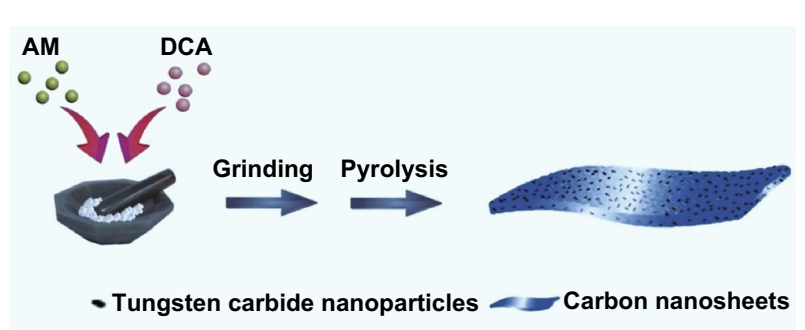

Fig. 1 Schematic illustration for solvent-free synthesis of different tungsten carbide/carbon composites using a $633 \mathrm{~nm}$ laser. X-ray photoelectron spectroscopy (XPS, Kratos, ULTRA AXIS DLD) was recorded to study the surface states with monochrome Al K $\alpha(1486.6 \mathrm{eV})$ radiation. Transmission electron microscopy (TEM) images were obtained on a Tecnai F20 instrument operating at an accelerating voltage of $200 \mathrm{kV}$. The thermogravimetric analysis was carried out on a SDT Q600 thermogravimetric analyzer (TGA) in the temperature range of room temperature to $800{ }^{\circ} \mathrm{C}$ at a heating rate of $10{ }^{\circ} \mathrm{C} \mathrm{min}{ }^{-1}$ under air atmosphere. The conductivities of WCC- $r$ were recorded on a four-probe resistivity meter (RTS-9, Guangzhou 4-probes Technology Co., Ltd, China). Before the measurement, 45 $w t \%$ of WCC- $r$ and $55 \mathrm{wt} \%$ of molten paraffin wax were adequately ground for about $30 \mathrm{~min}$ to obtain a uniform mixture, and then, the mixture was collected in a metallic disk with a diameter of $20 \mathrm{~mm}$ and a thickness of $0.30 \mathrm{~mm}$ for pressing into a circular sheet through a tablet machine. The relative complex permittivity and complex permeability in the frequency range of $2.0-18.0 \mathrm{GHz}$ were measured using an Agilent N5234A vector network analyzer (Agilent, USA) for the calculation of reflection loss characteristics. A sample containing $45 \mathrm{wt} \%$ of obtained composites was pressed into a ring with an outer diameter of $7.0 \mathrm{~mm}$, an inner diameter of $3.0 \mathrm{~mm}$, and a thickness of $2.0 \mathrm{~mm}$ for microwave measurement in which paraffin wax was used as the binder. The frequency span during the measurement was $0.08 \mathrm{GHz}$, and the symbols in the related curves were labeled at a given interval for clarification.

\section{Results and Discussion}

\subsection{Structure Characterization}

Figure 2 shows XRD patterns of tungsten carbide/carbon composites from different DCA/AM weight ratios. It can be seen that all samples exhibit three identical and well-resolved peaks at $37.0^{\circ}, 62.0^{\circ}$, and $74.2^{\circ}$. Tungsten carbide has long been known to have three crystalline structures, namely hexagonal WC phase, close-packed hexagonal $\mathrm{W}_{2} \mathrm{C}$ phase, and face-centered cubic $\mathrm{WC}_{1-x}$ phase [40]. The three peaks of these composites can be precisely matched with (111), (220), and (311) planes of $\mathrm{WC}_{1-x}[41,42]$, respectively, indicating that tungsten carbide mainly exists as $\mathrm{WC}_{1-x}$ phase in these composites. The obvious broadening of these peaks means that 


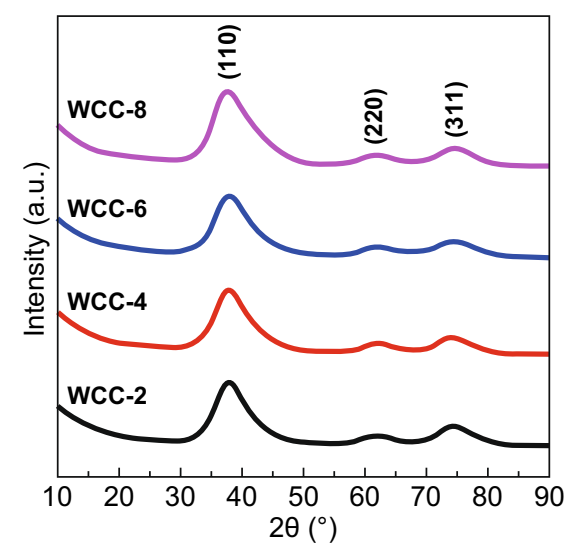

Fig. 2 XRD patterns of different tungsten carbide/carbon composites

$\mathrm{WC}_{1-x}$ in these composites has very small particle size. With these measured patterns, one can calculate that the average cell parameter $(a)$ of cubic $\mathrm{WC}_{1-x}$ nanoparticles herein is about $0.4223 \mathrm{~nm}$. Generally speaking, $\mathrm{WC}_{1-x}$ phase is usually generated under a carbon-rich condition [43]. Although the minimum weight ratio of DCA to AM is only 2.0 , the real $\mathrm{C} / \mathrm{W}$ molar ratio exceeds 10.0 , and thus, the formation of $\mathrm{WC}_{1-x}$ nanoparticles in these composites is understandable. Of note is that a larger dosage of DCA will not impact the crystalline phase of tungsten carbide nanoparticles any more. The large excess of DCA not only facilitates the formation of $\mathrm{WC}_{1-x}$ nanoparticles, but also produces some individual carbon components, while these individual carbon components fail to present any characteristic peaks due to their amorphous nature. Kurlov and Gusev ever investigated the phase transformation of tungsten carbide particles in detail [44], and they proposed that $a$ was a parabolic function of carbon content in $\mathrm{WC}_{1-x}$ particles, and their relationship could be described by Eq. 1 .

$a=0.4015+0.0481(1-x)-0.0236(1-x)^{2}$.

Based on Eq. 1, we get the value of $1-x$ is about 0.62 using 0.4223 , and thus, the specific expression of tungsten carbide nanoparticles in these composites should be $\mathrm{WC}_{0.62}$. Moreover, the survey of XPS spectra also detects very weak signals of $\mathrm{N}$ species at about $400 \mathrm{eV}$ (Fig. S1), suggesting that some $\mathrm{N}$ atoms in DCA are preserved in those individual carbon components. These $\mathrm{N}$ species can be considered as defective sites in carbon components, and thus, they can produce positive effect on the consumption of EM energy through polarization loss [11].

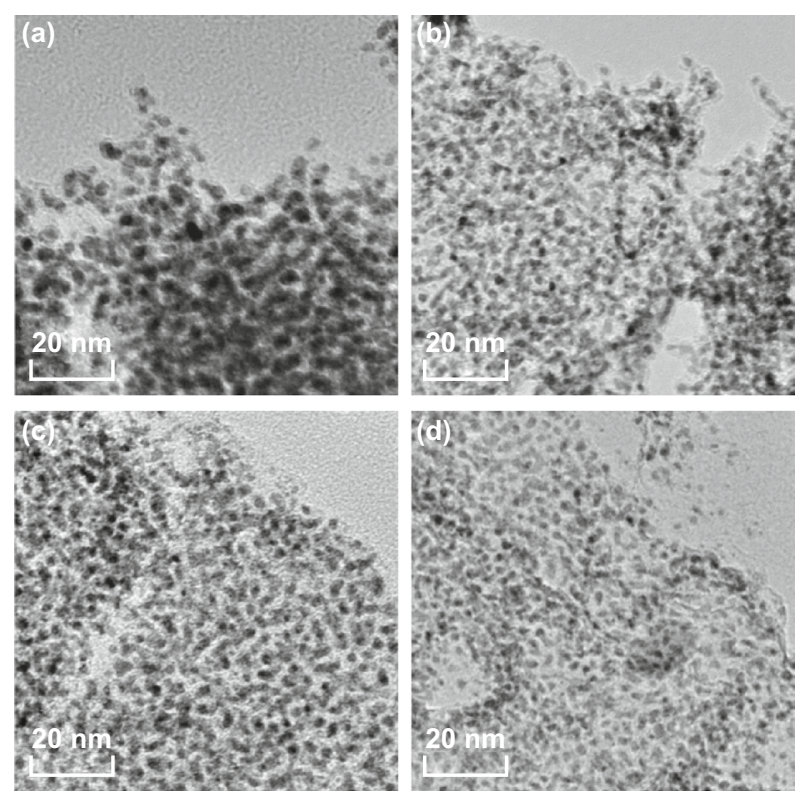

Fig. 3 TEM images of a WCC-2, b WCC-4, c WCC-6, and d WCC8

In order to better understand the microstructure of these tungsten carbide/carbon composites, TEM measurement is further carried out. As shown in Fig. S2, one can see that numerous ultrafine $\mathrm{WC}_{1-x}$ nanoparticles are uniformly dispersed on carbon nanosheets. It is believable that such good dispersion of $\mathrm{WC}_{1-x}$ nanoparticles on carbon nanosheets will create sufficient interfaces and be greatly helpful for the consumption of EM energy [45, 46]. With the increase in DCA/AM weight ratios, the deposition density of $\mathrm{WC}_{1-x}$ nanoparticles on carbon nanosheets is gradually decreased from WCC-2 to WCC-8, indicating that the chemical composition of these composites can be easily manipulated according to the dosages of AM and DCA. A closer inspection reveals that the average sizes of $\mathrm{WC}_{1-x}$ nanoparticles in these composites are all ca. 3-4 nm (Fig. 3). That is to say, the weight ratio of DCA to $\mathrm{AM}$ just regulates the relative contents of $\mathrm{WC}_{1-x}$ nanoparticles and carbon nanosheets rather than the crystalline structure and size of $\mathrm{WC}_{1-x}$ nanoparticles. In view of the fact that solvent-free synthesis has extremely high utilization efficiency of metal atoms and does not produce any wastewater, it is an absolutely green and sustainable strategy for tungsten carbide/carbon composites.

It is well known that EM properties of carbon-based composites are highly dependent on the relative content 
and graphitization degree of carbon components, especially for those without magnetic components [24, 47]. TG is an effective characterization method to determine carbon content in carbon-based composites, because carbon species will be totally removed at high temperature under air atmosphere [17, 48]. As shown in Fig. 4a, all four composites display quite similar TG curves that contain a weight increase region and a weight loss region. The former is attributed to the oxidation of $\mathrm{WC}_{1-x}$ nanoparticles, and the latter should be resulted from the combustion of carbon nanosheets. We characterize the residual powder after TG measurement and confirm that these composites will be completely transformed into $\mathrm{WO}_{3}$ (Fig. S3). In other words, not only carbon nanosheets but also carbon atoms in $\mathrm{WC}_{1-x}$ nanoparticles will be also removed simultaneously. Based on the possible expression of $\mathrm{WC}_{1-x}$ nanoparticles by XRD patterns, one can estimate the specific content of carbon nanosheets with Eq. 2:

$R \mathrm{wt} \%=(1-C \mathrm{wt} \%) \frac{M_{\mathrm{wO}_{3}}}{M_{\mathrm{WC}_{1-x}}}$

where $R \mathrm{wt} \%, \mathrm{C} \mathrm{wt} \%, M_{\mathrm{WO}_{3}}$, and $M_{\mathrm{wC}_{1-x}}$ are referred to remaining weight percentage, carbon nanosheets content, $\mathrm{WO}_{3}$ formula weight, and $\mathrm{WC}_{1-x}$ formula weight, respectively. The calculation results suggest that the specific contents of carbon nanosheets in WCC-2, WCC-4, WCC-6, and $\mathrm{WCC}-8$ are $21.3 \%, 29.3 \%, 32.6 \%$, and $35.8 \%$, respectively, which mean that the chemical composition of these composites can be finely regulated by the weight ratio of DCA to AM. However, due to the gap in formula weights between $\mathrm{C}$ and $\mathrm{WC}_{1-x}$, the large span of weight ratio from 2.0 to 8.0 only accounts the increase in the relative content of carbon nanosheets from 21.3 to $35.8 \%$. In addition, these composites give quite similar onset temperature (ca. $300{ }^{\circ} \mathrm{C}$ ) for weight increase, again verifying that the average sizes of $\mathrm{WC}_{1-x}$ nanoparticles in different composites are much pretty close, as indicated by TEM images (Fig. 2). WCC-8 has the lowest content of $\mathrm{WC}_{1-x}$ nanoparticles, while it promises the highest weight increase at about $480^{\circ} \mathrm{C}$. Obviously, the good dispersion of $\mathrm{WC}_{1-x}$ nanoparticles in WCC- 8 accounts for an intensive oxidation.

Figure 4b shows Raman spectra of tungsten carbide/ carbon composites from different DCA/AM weight ratios. As observed, all of them present two distinguishable bands at 1350 and $1600 \mathrm{~cm}^{-1}$, which are typical bands in carbon components and widely denoted as D band and G band, respectively [47, 49]. Ferrari and Robertson ever interpreted the evolution in Raman spectra from amorphous carbon to perfect graphite in detail [49]. They proposed that the change in the bonding state of carbon atoms could be linked with three features, $G$ band position, $G$ band profile, and the intensity ratio of $\mathrm{D}$ band to $\mathrm{G}$ band $\left(I_{\mathrm{D}} / I_{\mathrm{G}}\right)$. During the stage from amorphous carbon to nanocrystalline graphite, the position of $\mathrm{G}$ band gradually shifts from 1510 to $1600 \mathrm{~cm}^{-1}$ and the profile of $\mathrm{G}$ band becomes relatively narrow, and meanwhile, an incremental $I_{\mathrm{D}} / I_{\mathrm{G}}$ value from 0.25 to 2.0 can be also detected. These changes are attributed to the decreased disorders in bond angle and bond bending, as well as the tightened vibrational density of states. However, these tiny crystalline domains are very difficult to detect, even by high-resolution TEM $[50,51]$. When nanocrystalline graphite is further transformed into perfect graphite, all these changes will be inverse. In our case, the positions of these tungsten carbide/carbon composites are all located at $1600 \mathrm{~cm}^{-1}$, while the local amplification reveals that the values of full-width half-maximum in G band for WCC-2,
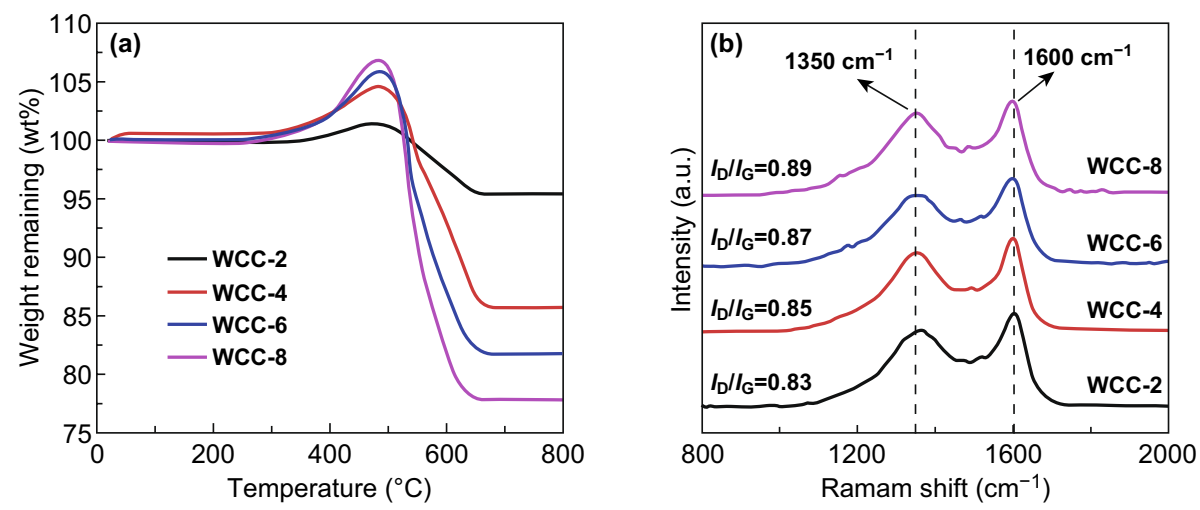

Fig. 4 a TG curves and b Raman spectra of different tungsten carbide/carbon composites 
WCC-4, WCC-6, and WCC-8 are 110, 106, 96, and $88 \mathrm{~cm}^{-1}$, respectively (Fig. S4). At the same time, one can also find that $I_{\mathrm{D}} / I_{\mathrm{G}}$ values slowly increase from 0.83 to 0.89 . These phenomena suggest the formation of nanocrystalline graphite domains in amorphous carbon nanosheets, that is, more $\mathrm{W}$ species may decrease the relative graphitization degree of carbon nanosheets to some extent.

\subsection{Dielectric Property}

Relative complex permittivity $\left(\varepsilon_{\mathrm{r}}=\varepsilon_{\mathrm{r}}{ }^{\prime}-\mathrm{j} \varepsilon_{\mathrm{r}}{ }^{\prime \prime}\right)$ and complex permeability $\left(\mu_{\mathrm{r}}=\mu_{\mathrm{r}}{ }^{\prime}-\mathrm{j} \mu_{\mathrm{r}}{ }^{\prime \prime}\right)$ are two extremely important parameters to determine the performance of MAMs [17, 52]. Herein, all tungsten carbide/carbon composites are free of any magnetic components, and thus, their real and imaginary parts of complex permeability are constant at 1 and 0 , respectively (Fig. S5). That is to say, dielectric loss is the only pathway to attenuate incident EM energy [24, 36]. Figure 5 shows $\varepsilon_{\mathrm{r}}{ }^{\prime}$ and $\varepsilon_{\mathrm{r}}{ }^{\prime}$ curves of different tungsten carbide/ carbon composites in the frequency range of $2.0-18.0 \mathrm{GHz}$. Among them, WCC-2 has the lowest $\varepsilon_{\mathrm{r}}{ }^{\prime}$ and $\varepsilon_{\mathrm{r}}{ }^{\prime \prime}$ values, which are insusceptible to the frequency and almost constant at 4.35 and 0.24 , respectively. When more carbon nanosheets are introduced, both $\varepsilon_{\mathrm{r}}{ }^{\prime}$ and $\varepsilon_{\mathrm{r}}{ }^{\prime \prime}$ values are significantly increased. Especially for WCC-6 and WCC-8, they not only have larger $\varepsilon_{\mathrm{r}}{ }^{\prime}$ and $\varepsilon_{\mathrm{r}}{ }^{\prime \prime}$ values, but also present typical frequency dispersion behaviors. For example, $\varepsilon_{\mathrm{r}}{ }^{\prime}$ and $\varepsilon_{\mathrm{r}}{ }^{\prime \prime}$ values of WCC-6 gradually decrease from 18.87 and 12.60 at $2.0 \mathrm{GHz}$ to 10.87 and 4.25 at $18.0 \mathrm{GHz}$, respectively, and $\varepsilon_{\mathrm{r}}{ }^{\prime}$ and $\varepsilon_{\mathrm{r}}{ }^{\prime \prime}$ values of WCC-8 gradually decrease from 29.84 and 21.09 at $2.0 \mathrm{GHz}$ to 13.33 and 8.43 at $18.0 \mathrm{GHz}$, respectively. Dielectric loss tangent $\left(\tan \delta_{\mathrm{e}}=\varepsilon_{\mathrm{r}}{ }^{\prime \prime} / \varepsilon_{\mathrm{r}}{ }^{\prime}\right)$ further demonstrates that the dielectric loss ability of these composites is continuously enhanced with the increase in carbon nanosheets (Fig. S6).

It is believable that this enhancement should be associated with the following two aspects: conductive loss and polarization loss [53-55]. Conductive loss is always generated by some residual carriers in dielectric medium, and their directional movement under an applied electric field will convert electric energy into heat energy [56]. In general, carbon component has better conductivity than $\mathrm{WC}_{1-x}$ nanoparticles [57], and thus, the increase in carbon nanosheets can improve the conductivity of tungsten carbide/carbon composites. Four-probe measurement also reveals that the conductivities of WCC-2, WWC-4, WCC-6, and WCC-8 in wax are $4.21 \times 10^{-5}, 2.03 \times 10^{-4}, 8.71 \times 10^{-4}$, and $5.29 \times 10^{-3} \mathrm{~S}$ $\mathrm{m}^{-1}$, respectively. Although the relative content of carbon nanosheets is not remarkably increased, its lower density as compared with $\mathrm{WC}_{1-x}$ nanoparticles may promise a larger volume fraction, resulting in the formation of conductive networks in wax matrix and considerable contribution to conductive loss.

Compared to conductive loss, polarization loss has diversified modes, i.e., electronic polarization, ionic polarization, dipole orientation polarization, and interfacial polarization $[54,55]$. However, electronic polarization and ionic polarization are widely accepted to be inactive for the dissipation of EM energy in gigahertz range because their relaxation time is too short $\left(10^{-12}-10^{-16} \mathrm{~s}\right)[54,55]$. Therefore, dipole orientation polarization and interfacial polarization are the two modes that can be responsible for energy consumption under
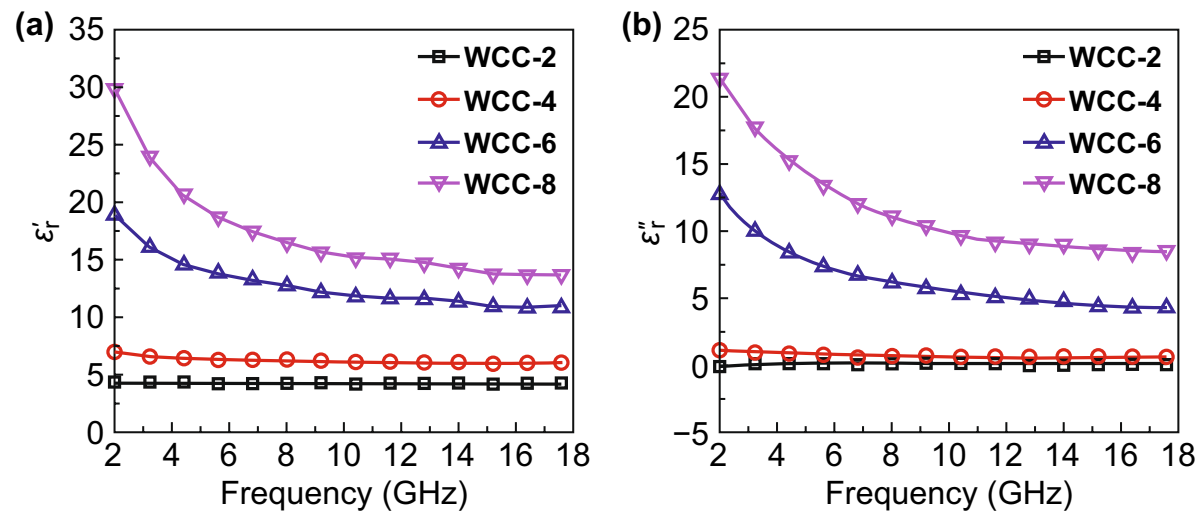

Fig. 5 a Real parts and $\mathbf{b}$ imaginary parts of relative complex permittivity of different tungsten carbide/carbon composites 
current conditions. Dipole orientation polarization usually comes from the hysteretic reorientation of dipoles along with an applied electric field [58, 59]. In our case, there are two kinds of dipole orientation polarization relaxations. One is from intrinsic dipoles in $\mathrm{WC}_{1-x}$ nanoparticles, and the other is from bound charges in residual functional groups and defective sites ( $\mathrm{N}$ sites) in carbon nanosheets. With the increase in carbon nanosheets, the number of intrinsic dipoles in $\mathrm{WC}_{1-x}$ nanoparticles will decrease, while bound charges in carbon nanosheets may make up the loss of dipoles, and thus, the contribution of dipole orientation polarization will not significantly change.

As for interfacial polarization, it mainly depends on the asymmetrical accumulation of space charges at heterogeneous interfaces, which can generate an electric dipole moment to drive energy consumption [45, 54]. That is to say, more heterogeneous interfaces can produce more powerful interfacial polarization. The increase in carbon nanosheets alleviates the aggregation of $\mathrm{WC}_{1-x}$ nanoparticles effectively (Fig. S2), and thus, there will be more interfaces between $\mathrm{WC}_{1-x}$ nanoparticles and carbon nanosheets from WCC-2 to WCC-8, accounting for the enhancement of interfacial polarization. Actually, when polarization loss works for the dissipation of EM energy, frequency dispersion behaviors may occur in $\varepsilon_{\mathrm{r}}{ }^{\prime}$ and $\varepsilon_{\mathrm{r}}{ }^{\prime \prime}$ curves [36, 58], as indicated in Fig. 5. Meanwhile, the contribution of polarization loss can also be witnessed by several semicircles in the curves of $\varepsilon_{\mathrm{r}}^{\prime \prime}$ versus $\varepsilon_{\mathrm{r}}^{\prime}$ (Fig. S7), where each semicircle (denoted as Cole-Cole semicircle) corresponds to one polarization relaxation according to Debye theory [47, 55]. However, Cole-Cole semicircles just describe the number of relaxation processes, but not their intensities. The less semicircles in WCC- 6 and WCC- 8 may be attributed to the fact that the decrease in the relative content of $\mathrm{WC}_{1-x}$ nanoparticles weakens their intrinsic dipole orientation polarization. From the above analyses, it can be concluded that the overall dielectric loss is the sum of conductive loss, dipole orientation polarization, and interfacial polarization, while conductive loss and interfacial polarization play two more progressive roles to reinforce dielectric loss from WCC-2 to WCC-8.

\subsection{Microwave Absorption and Mechanism}

Based on transmission line theory [59], microwave absorption performance of tungsten carbide/carbon composites can be calculated with these measured EM parameters by Eqs. 3 and 4 ,

$\operatorname{RL}(\mathrm{dB})=20 \log \left|\frac{Z_{\text {in }}-1}{Z_{\text {in }}+1}\right|$

$Z_{\text {in }}=\sqrt{\frac{\mu_{r}}{\varepsilon_{r}}} \tanh \left[j\left(\frac{2 \pi}{c}\right) f d \sqrt{\mu_{\mathrm{r}} \cdot \varepsilon_{\mathrm{r}}}\right]$

where $c, f$, and $d$ correspond the velocity of EM wave in free space (i.e., $3 \times 10^{8} \mathrm{~m} / \mathrm{s}$ ), the frequency of EM wave, and the thickness of MAMs, respectively. Figure 6 plots the threedimensional RL maps of four tungsten carbide/carbon composites by employing $f(2.0-18.0 \mathrm{GHz})$ and $d(1.0-5.0 \mathrm{~mm})$ as two independent variables. As observed, WCC-2 fails to produce any effective microwave absorption (Fig. 6a) due to its feeble dielectric loss ability and inactive magnetic loss ability (Figs. 5 and S5). Although the increase in dielectric loss ability brings more or less improvement in the performance of WCC-4 (Fig. 6b), its minimum RL intensity is still larger than $-10 \mathrm{~dB}$, a commonly indicative value for qualified microwave absorption that equals to $90 \%$ absorption efficiency [47, 59]. In contrast, WCC-6 displays much better microwave absorption performance (Fig. 6c), whose strongest RL intensity can reach up to $-55.6 \mathrm{~dB}(d=1.34 \mathrm{~mm}$, $f=17.5 \mathrm{GHz}$ ) and qualified absorption bandwidth covers the frequency range of 3.6-18.0 GHz. The cutoff of RL value in Fig. $6 \mathrm{c}$ is artificially set at $-30.0 \mathrm{~dB}$ for a clear comparison, while the corresponding RL curve with the strongest absorption can be identified in Fig. S8. With further increasing carbon content, WCC- 8 does not promise an expected enhancement in microwave absorption (Fig. 6d), and instead, its strongest RL intensity and qualified absorption bandwidth fall back to $-11.3 \mathrm{~dB}(d=1.20 \mathrm{~mm}, f=18.0 \mathrm{GHz})$ and $7.0 \mathrm{GHz}(11.0-18.0 \mathrm{GHz})$. It is undoubted that WCC-6 is the best candidate among these tungsten carbide/carbon composites, no matter from RL intensity or qualified absorption bandwidth. In order to validate the performance of WCC-6 with those reported carbide/carbon composites, we summarize their RL characteristics in some appointed frequency intervals (Table 1) [28-31, 34-37, 60-63]. In general, researchers pay more attention to qualified absorption bandwidth, because there is no essential difference when RL intensity exceeds $-10.0 \mathrm{~dB}$. One can easily find that WCC-6 indeed possess top-level microwave absorption performance in different frequency ranges, and it can even realize equivalent bandwidth with less $d$ value. Simple synthesis and good performance offer a good platform for the practical application of such tungsten carbide/carbon composites.

It has to mention that there is a common relationship between the thickness and the frequency corresponding 

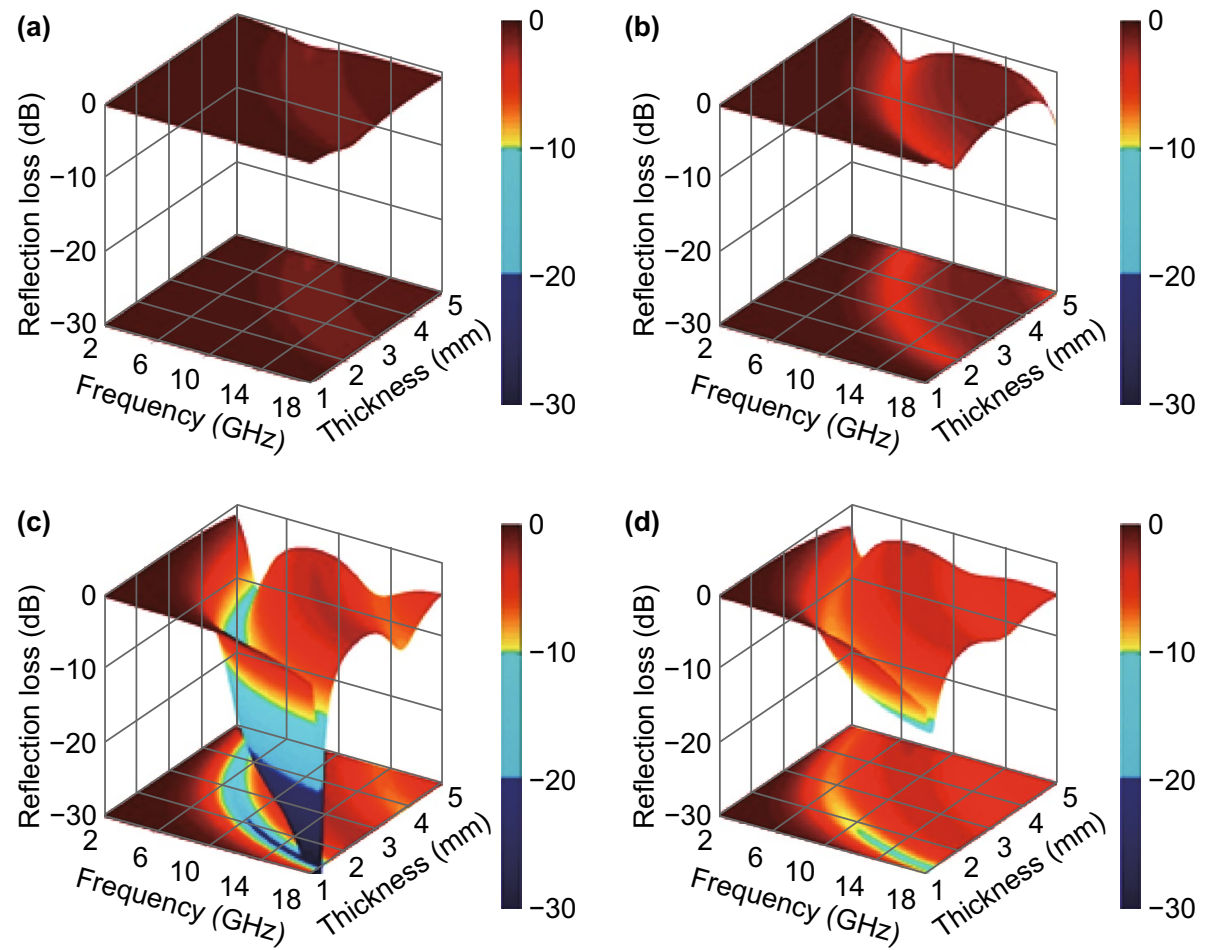

Fig. 6 Three-dimensional RL maps of a WCC-2, b WCC-4, c WCC-6, and d WCC-8

to minimum RL peaks in all these composites, i.e., a large $d$ value can induce the shift of absorption peak to lowfrequency region (Fig. 6). This phenomenon implies that incident EM waves may be attenuated based on a quarterwavelength $(\lambda / 4)$ matching model. If this model works, the incident EM waves and reflected waves from a metal-backed layer with a phase difference at $180^{\circ}$ will offset each other through destructive interference and realize the consumption of EM energy. In that case, the thickness of MAMs and the frequency of minimum absorption peak may satisfy Eq. 5 [33, 64]:

$t_{\mathrm{m}}=\frac{n}{4} \lambda_{\mathrm{m}}=\frac{n c}{4 f_{\mathrm{m}} \sqrt{\left|\varepsilon_{\mathrm{r}} \cdot \mu_{\mathrm{r}}\right|}}(n=1,3,5 \ldots)$

where $t_{\mathrm{m}}$ and $f_{\mathrm{m}}$ are theoretical thickness and frequency, respectively. As shown in Fig. S9, the specific $d$ values (green stars) of these tungsten carbide/carbon composites are exactly dispersed on the curves of $t_{\mathrm{m}}$ versus $f_{\mathrm{m}}$ (orange lines), confirming that EM dissipation in these composites is in good agreement with the quarter-wavelength matching model, that is, a strong EM extinction effect will occur at the specific values of $d$ and $f$.
It is widely accepted that RL characteristics of MAMs are highly dependent on their overall attenuation ability from both dielectric loss and magnetic loss [59, 65]. In our case, magnetic components are excluded in all these tungsten carbide/carbon composites, and thus, the attenuation constant $(\alpha)$ is determined by their dielectric loss alone. As confirmed in Fig. S10, all these composites give monotonously increased $\alpha$ values in the frequency range of $2.0-18.0 \mathrm{GHz}$, and the order of $\alpha$ value at a specific point is exactly consistent with that of dielectric loss tangent (Fig. S6). However, WCC-8 with the largest $\alpha$ value is incapable of generating the best microwave absorption performance (Fig. 6). This is because RL characteristics are also related to another important factor, impedance matching degree [66]. If the impedance of MAMs is poorly matched with that of free space, a strong reflection of incident EM waves will occur at the interface. As a result, no matter how strong their intrinsic attenuation ability is, they will not bring desirable microwave absorption performance. The ideal condition for perfect impedance matching requires that MAMs have identical $\varepsilon_{\mathrm{r}}$ and $\mu_{\mathrm{r}}$, while it is impossible except in a wave-transparent 


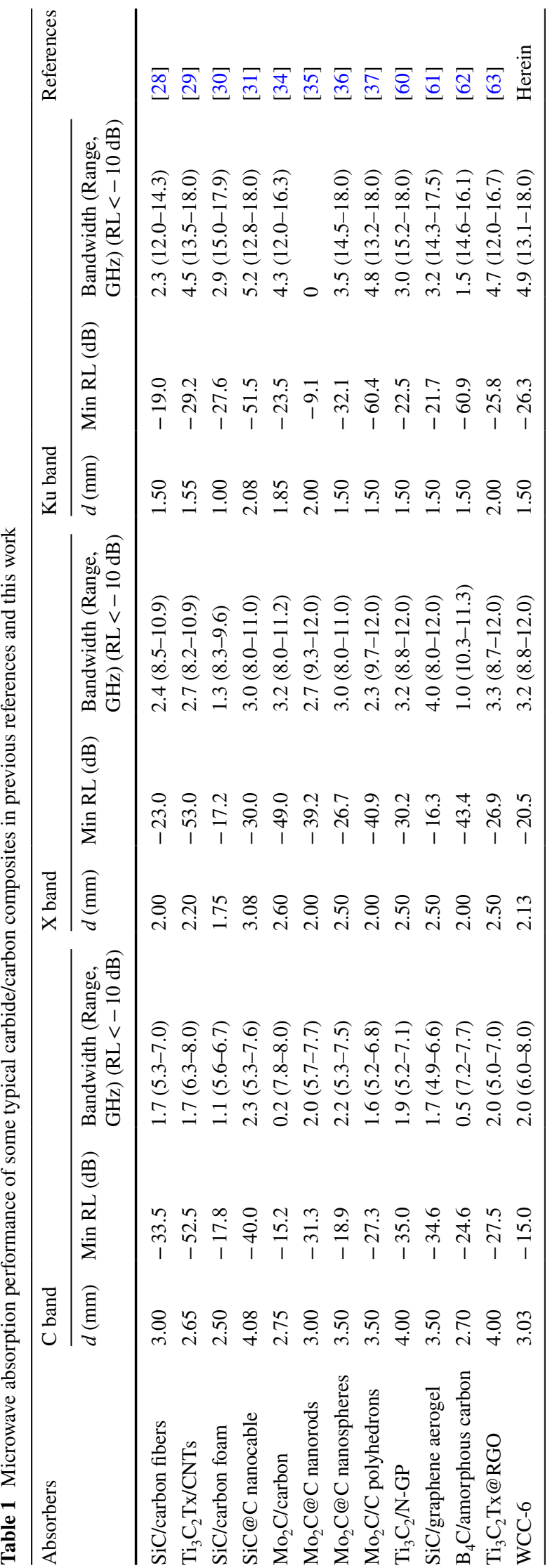

medium that has negligible dielectric loss and magnetic loss $[67,68]$. In common media dominated by dielectric loss, $\varepsilon_{\mathrm{r}}$ is usually much larger than $\mu_{\mathrm{r}}$, which means that the gap between $\varepsilon_{\mathrm{r}}$ and $\mu_{\mathrm{r}}$ should be tailored within a rational range to fulfill good attenuation ability and impedance matching simultaneously $[37,69]$. We further employ a delta-function method to visibly illustrate the difference of impedance matching degree in WCC- 6 and WCC-8 (Fig. 7). In this figure, the modules of delta values at different frequency points and absorber thickness can be calculated by Eqs. 6-8 [70],

$$
\begin{aligned}
& |\Delta|=\left|\sinh ^{2}(K f d)-M\right| \\
& K=\frac{4 \pi \sqrt{\mu_{\mathrm{r}}^{\prime} \cdot \varepsilon_{\mathrm{r}}^{\prime}} \cdot \sin \frac{\delta_{\mathrm{e}}+\delta_{\mathrm{m}}}{2}}{c \cdot \cos \delta_{\mathrm{e}} \cdot \cos \delta_{\mathrm{m}}} \\
& M=\frac{4 \mu_{\mathrm{r}}^{\prime} \cdot \cos \delta_{\mathrm{e}} \cdot \varepsilon_{\mathrm{r}}^{\prime} \cdot \cos \delta_{\mathrm{m}}}{\left(\mu_{\mathrm{r}}^{\prime} \cdot \cos \delta_{\mathrm{e}}-\varepsilon_{\mathrm{r}}^{\prime} \cdot \cos \delta_{\mathrm{m}}\right)+\left[\tan \left(\frac{\delta_{\mathrm{m}}}{2}-\frac{\delta_{\mathrm{e}}}{2}\right)\right]^{2} \cdot\left(\mu_{\mathrm{r}}^{\prime} \cdot \cos \delta_{\mathrm{e}}+\varepsilon_{\mathrm{r}}^{\prime} \cdot \cos \delta_{\mathrm{m}}\right)^{2}} .
\end{aligned}
$$

The smaller the modules of delta values are, the better impedance matching degree is achieved, and the threshold for good impedance matching is usually accepted as $|\Delta| \leq 0.4[69,70]$. It is clear that WCC-6 possesses a good matching region with the coverage ratio at about $39.1 \%$, while the coverage of matching region in WCC-8 drastically shrinks back to $13.2 \%$. These results explain that the recession of microwave absorption performance in WCC-8 is mainly attributed to its deteriorative impedance matching degree. To support this viewpoint, we further raise the DCA/AM weight ratio to 10.0 , and the relative complex permittivity and dielectric loss tangent of WCC-10 are further enhanced as compared with WCC-8 (Fig. S11a, b). It is unfortunate that the impedance matching degree is further deteriorated, whose coverage of matching region is only $3.1 \%$ (Fig. S11c), again verifying that the overlarge gap between relative complex permittivity and complex permeability is not beneficial to good impedance matching. Correspondingly, WCC-10 cannot produce good microwave absorption performance due to strong reflection induced by such poor impedance matching (Fig. S11d). That is to say, the pursuit of strong dielectric loss ability is not certainly favorable for RL characteristics, and the impedance matching should be also taken into account seriously. 

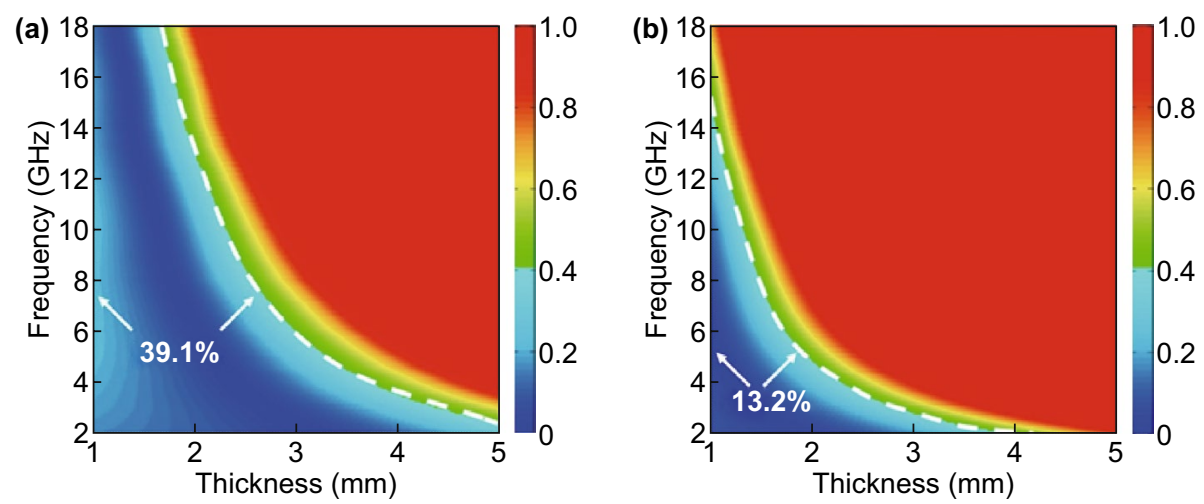

Fig. 7 Delta maps of a WCC-6 and b WCC-8 with different absorber thickness in the frequency range of 2.0-18.0 GHz

\section{Conclusions}

Tungsten carbide/carbon composites have been successfully prepared with the solid mixture of dicyandiamide (DCA) and ammonium metatungstate (AM) as a precursor. Ultrafine cubic tungsten carbide nanoparticles in situ formed during high-temperature pyrolysis are homogeneously dispersed on carbon nanosheets. It is found that the weight ratio of DCA to AM plays an important role in determining the relative contents of carbon nanosheets and $\mathrm{WC}_{1-x}$ nanoparticles, as well as their dielectric properties. The composite WCC-6 (DCA:AM=6:1) exhibits good microwave absorption, whose strongest reflection loss reaches up to $-55.6 \mathrm{~dB}$ and qualified absorption bandwidth covers the frequency range of 3.6-18.0 GHz. Such a performance benefits from its decent attenuation ability and desirable impedance matching and is indeed superior to many conventional carbides/carbon composites. It is believable that these results are not only helpful for the preparation of high-performance microwave-absorbing materials in a green and sustainable way, but also inspire the development of functional carbide/carbon composites in other fields.

Acknowledgements This work is supported by National Natural Science Foundation of China (21676065 and 21776053).

Open Access This article is licensed under a Creative Commons Attribution 4.0 International License, which permits use, sharing, adaptation, distribution and reproduction in any medium or format, as long as you give appropriate credit to the original author(s) and the source, provide a link to the Creative Commons licence, and indicate if changes were made. The images or other third party material in this article are included in the article's Creative Commons licence, unless indicated otherwise in a credit line to the material. If material is not included in the article's Creative Commons licence and your intended use is not permitted by statutory regulation or exceeds the permitted use, you will need to obtain permission directly from the copyright holder. To view a copy of this licence, visit http://creativecommons.org/licenses/by/4.0/.

Electronic supplementary material The online version of this article (https://doi.org/10.1007/s40820-020-00491-5) contains supplementary material, which is available to authorized users.

\section{References}

1. J. Liu, H. Zhang, R. Sun, Y. Liu, Z. Liu, A. Zhou, Z. Yu, Hydrophobic, flexible, and lightweight MXene foams for high-performance electromagnetic-interference shielding. Adv. Mater. 29, 1702367 (2017). https://doi.org/10.1002/ adma. 201702367

2. A. Singh, M. Mishra, P. Sambyal, B. Gupta, B. Singh, A. Chandra, S. Dhawan, Encapsulation of $\gamma-\mathrm{Fe}_{2} \mathrm{O}_{3}$ decorated reduced graphene oxide in polyaniline core-shell tubes as an exceptional tracker for electromagnetic environmental pollution. J. Mater. Chem. A 2, 3581-3593 (2014). https://doi. org/10.1039/c3ta14212d

3. C. Wang, V. Murugadoss, J. Kong, Z. He, X. Mai et al., Overview of carbon nanostructures and nanocomposites for electromagnetic wave shielding. Carbon 140, 696-733 (2018). https ://doi.org/10.1016/j.carbon.2018.09.006

4. L. Huang, Y. Duan, X. Dai, Y. Zeng, G. Ma et al., Bioinspired metamaterials: multibands electromagnetic wave adaptability and hydrophobic characteristics. Small 15, 1902730 (2019). https://doi.org/10.1002/smll.201902730

5. H. Abbasi, M. Antunes, J. Velasco, Recent advances in carbon-based polymer nanocomposites for electromagnetic 
interference shielding. Prog. Mater Sci. 103, 313 (2019). https ://doi.org/10.1016/j.pmatsci.2019.02.003

6. X. Wang, J. Shu, W. Cao, M. Zhang, J. Yuan, M. Cao, Ecomimetic nanoarchitecture for green EMI shielding. Chem. Eng. J. 369, 1068-1077 (2019). https://doi.org/10.1016/j. cej.2019.03.164

7. X. Sun, M. Yang, S. Yang, S. Wang, W. Yin, R. Che, Y. $\mathrm{Li}$, Ultrabroad band microwave absorption of carbonized waxberry with hierarchical structure. Small 15, 1902974 (2019). https://doi.org/10.1002/small.201902974

8. Y. Zhang, Y. Huang, T. Zhang, H. Chang, P. Xiao et al., Broadband and tunable high-performance microwave absorption of an ultralight and highly compressible graphene foam. Adv. Mater. 27, 2049-2053 (2015). https://doi.org/10.1002/ adma.201405788

9. H. Zhao, Y. Cheng, W. Liu, L. Yang, B. Zhang et al., Biomass-derived porous carbon-based nanostructures for microwave absorption. Nano-Micro Lett. 11, 24 (2019). https://doi. org/10.1007/s40820-019-0255-3

10. R. Qiang, Y. Du, Y. Wang, N. Wang, C. Tian et al., Rational design of yolk-shell C@C microspheres for the effective enhancement in microwave absorption. Carbon 98, 599-606 (2016). https://doi.org/10.1016/j.carbon.2015.11.054

11. P. Liu, Y. Zhang, J. Yan, Y. Huang, L. Xia, Z. Guang, Synthesis of lightweight $\mathrm{N}$-doped graphene foams with open reticular structure for high-efficiency electromagnetic wave absorption. Chem. Eng. J. 368, 285-298 (2019). https://doi.org/10.1016/j. cej.2019.02.193

12. Y. Wang, Y. Du, R. Qiang, C. Tian, P. Xu, X. Han, Interfacially engineered sandwich-like $\mathrm{rGO}$ /carbon microspheres/ rGO composite as an efficient and durable microwave absorber. Adv. Mater. Interfaces 3, 1500684 (2016). https:// doi.org/10.1002/admi.201500684

13. M. Cao, J. Yang, W. Song, D. Zhang, B. Wen et al., Ferroferric oxide/multiwalled carbon nanotube vs polyaniline/ferroferric oxide/multiwalled carbon nanotube multiheterostructures for highly effective microwave absorption. ACS Appl. Mater. Interfaces. 4, 6949-6956 (2012). https://doi.org/10.1021/ am3021069

14. W. Liu, Q. Shao, G. Ji, X. Liang, Y. Cheng, B. Quan, Y. Du, Metal-organic-frameworks derived porous carbon-wrapped Ni composites with optimized impedance matching as excellent lightweight electromagnetic wave absorber. Chem. Eng. J. 313, 734-744 (2017). https://doi.org/10.1016/j.cej.2016.12.117

15. I. Arief, S. Biswas, S. Bose, FeCo-anchored reduced graphene oxide framework-based soft composites containing carbon nanotubes as highly efficient microwave absorbers with excellent heat dissipation ability. ACS Appl. Mater. Interfaces. 9, 19202-19214 (2017). https://doi.org/10.1021/acsami.7b04053

16. X. Liu, X. Cui, Y. Chen, X. Zhang, R. Yu, G. Wang, H. Ma, Modulation of electromagnetic wave absorption by carbon shell thickness in carbon encapsulated magnetite nanospindles-poly(vinylidene fluoride) composites. Carbon 95, 870878 (2015). https://doi.org/10.1016/j.carbon.2015.09.036

17. D. Liu, R. Qiang, Y. Du, Y. Wang, C. Tian, X. Han, Prussian blue analogues derived magnetic FeCo alloy/carbon composites with tunable chemical composition and enhanced microwave absorption. J. Colloid Interfaces Sci. 514, 10-20 (2018). https://doi.org/10.1016/j.jcis.2017.12.013

18. S. Sankaran, K. Deshmukh, M. Ahamed, S. Khadheer Pasha, Recent advances in electromagnetic interference shielding properties of metal and carbon filler reinforced flexible polymer composites: a review. Compos. Part A 114, 49-71 (2018). https://doi.org/10.1016/j.compositesa.2018.08.006

19. Y. Wang, W. Wang, J. Sun, C. Sun, Y. Feng, Z. Li, Microwavebased preparation and characterization of Fe-cored carbon nanocapsules with novel stability and super electromagnetic wave absorption performance. Carbon 135, 1-11 (2018). https ://doi.org/10.1016/j.carbon.2018.04.026

20. X. Yang, Y. Duan, Y. Zeng, H. Pang, G. Ma, X. Dai, Experimental and theoretical evidence for temperature driving an electric-magnetic complementary effect in magnetic microwave absorbing materials. J. Mater. Chem. C 8, 1583-1590 (2020). https://doi.org/10.1039/c9tc06551b

21. X. Zhang, G. Wang, Y. Wei, L. Guo, M. Cao, Polymer-composite with high dielectric constant and enhanced absorption properties based on graphene-CuS nanocomposites and polyvinylidene fluoride. J. Mater. Chem. A 1, 12115-12122 (2013). https://doi.org/10.1039/c3ta12451g

22. H. Chen, Z. Huang, Y. Huang, Y. Zhang, Z. Ge et al., Synergistically assembled MWCNT/graphene foam with highly efficient microwave absorption in both $\mathrm{C}$ and $\mathrm{X}$ bands. Carbon 124, 506-514 (2017). https://doi.org/10.1016/j.carbo n.2017.09.007

23. P. Liu, Y. Huang, J. Yan, Y. Zhao, Magnetic graphene@ PANI@ porous $\mathrm{TiO}_{2}$ ternary composites for high-performance electromagnetic wave absorption. J. Mater. Chem. C 4, 63626370 (2016). https://doi.org/10.1039/c6tc01718e

24. L. Cui, C. Tian, L. Tang, X. Han, Y. Wang et al., Space-confined synthesis of core-shell $\mathrm{BaTiO}_{3} @$ carbon microspheres as a high-performance binary dielectric system for microwave absorption. ACS Appl. Mater. Interfaces. 11, 31182-31190 (2019). https://doi.org/10.1021/acsami.9b09779

25. S. Chiu, H. Yu, Y. Li, High electromagnetic wave absorption performance of silicon carbide nanowires in the gigahertz range. J. Phys. Chem. C 114, 1947-1952 (2010). https://doi. org/10.1021/jp905127t

26. M. Han, X. Yin, H. Wu, Z. Hou, C. Song et al., $\mathrm{Ti}_{3} \mathrm{C}_{2}$ MXenes with modified surface for high-performance electromagnetic absorption and shielding in the X-band. ACS Appl. Mater. Interfaces. 8, 21011-21019 (2016). https://doi.org/10.1021/ acsami.6b06455

27. X. Li, X. Yin, C. Song, M. Han, H. Xu et al., Self-assembly core-shell graphene-bridged hollow MXenes spheres 3D foam with ultrahigh specific EM absorption performance. Adv. Funct. Mater. 41, 1803938 (2018). https://doi.org/10.1002/ adfm. 201803938

28. L. Yan, C. Hong, B. Sun, G. Zhao, Y. Cheng et al., In situ growth of core-sheath heterostructural $\mathrm{SiC}$ nanowire arrays on carbon fibers and enhanced electromagnetic wave absorption performance. ACS Appl. Mater. Interfaces. 9, 6320-6331 (2017). https://doi.org/10.1021/acsami.6b15795 
29. X. Li, X. Yin, M. Han, C. Song, H. Xu et al., $\mathrm{Ti}_{3} \mathrm{C}_{2}$ MXenes modified with: in situ grown carbon nanotubes for enhanced electromagnetic wave absorption properties. J. Mater. Chem. C 5, 4068-4074 (2017). https://doi.org/10.1039/c6tc05226f

30. X. Ye, Z. Chen, S. Ai, B. Hou, J. Zhang et al., Effects of SiC coating on microwave absorption of novel three-dimensional reticulated $\mathrm{SiC} /$ porous carbon foam. Ceram. Int. 45, 86608668 (2019). https://doi.org/10.1016/j.ceramint.2019.01.186

31. M. Zhang, H. Lin, S. Ding, T. Wang, Z. Li et al., Net-like SiC@C coaxial nanocable towards superior lightweight and broadband microwave absorber. Compos. Part B 179, 107525 (2019). https://doi.org/10.1016/j.compositesb.2019.107525

32. H. Baskey, S. Singh, M. Akhtar, K. Kar, Investigation on the dielectric properties of exfoliated graphite silicon carbide nanocomposites and their absorbing capability for the microwave radiation. IEEE Trans. Nanotechnol. 16, 453-461 (2017). https://doi.org/10.1109/tnano.2017.2682121

33. Y. Wang, X. Li, X. Han, P. Xu, L. Cui et al., Ternary $\mathrm{Mo}_{2} \mathrm{C} /$ $\mathrm{Co} / \mathrm{C}$ composites with enhanced electromagnetic waves absorption. Chem. Eng. J. 387, 124159 (2020). https://doi. org/10.1016/j.cej.2020.124159

34. S. Dai, Y. Cheng, B. Quan, X. Liang, W. Liu et al., Porous carbon-based $\mathrm{Mo}_{2} \mathrm{C}$ nanocomposites as excellent microwave absorber: a new exploration. Nanoscale 10, 6945-6953 (2018). https://doi.org/10.1039/c8nr01244j

35. C. Li, X. Shen, R. Ding, G. Wang, Excellent microwave absorption properties based on a composite of one dimensional $\mathrm{Mo}_{2} \mathrm{C} @ \mathrm{C}$ nanorods and a PVDF matrix. RSC Adv. 9, 21243-21248 (2019). https://doi.org/10.1039/c9ra03362a

36. Y. Wang, X. Han, P. Xu, D. Liu, L. Cui, H. Zhao, Y. Du, Synthesis of pomegranate-like $\mathrm{Mo}_{2} \mathrm{C} @ \mathrm{C}$ nanospheres for highly efficient microwave absorption. Chem. Eng. J. 372, 312-320 (2019). https://doi.org/10.1016/j.cej.2019.04.153

37. Y. Wang, C. Li, X. Han, D. Liu, H. Zhao et al., Ultrasmall $\mathrm{Mo}_{2} \mathrm{C}$ nanoparticle-decorated carbon polyhedrons for enhanced microwave absorption. ACS Appl. Nano Mater. 1, 5366-5376 (2018). https://doi.org/10.1021/acsanm.8b01479

38. Q. Wu, X. Meng, X. Gao, F. Xiao, Solvent-free synthesis of zeolites: mechanism and utility. Acc. Chem. Res. 51, 13961403 (2018). https://doi.org/10.1021/acs.accounts.8b00057

39. C. Schneidermann, N. Jäckel, S. Oswald, L. Giebeler, V. Presser, L. Borchardt, Solvent-free mechanochemical synthesis of nitrogen-doped nanoporous carbon for electrochemical energy storage. Chemsuschem 10, 2416-2424 (2017). https:// doi.org/10.1002/cssc.201700459

40. A. Voevodin, J. O’Neill, S. Prasad, J. Zabinski, Nanocrystalline WC and WC/a-C composite coatings produced from intersected plasma fluxes at low deposition temperatures. J. Vac. Sci. Technol., A 17, 986-992 (1999). https://doi. org/10.1116/1.581674

41. J. Kim, J. Jang, Y. Lee, Y. Kwon, Enhancement of electrocatalytic activity of platinum for hydrogen oxidation reaction by sonochemically synthesized $\mathrm{WC}_{1-x}$ nanoparticles. J. Power Sources 193, 441-446 (2009). https://doi.org/10.1016/j.jpows our.2009.03.070
42. I. Kim, S. Park, D. Kim, Carbon-encapsulated multi-phase nanocomposite of $\mathrm{W}_{2} \mathrm{C} @ \mathrm{WC}_{1-x}$ as a highly active and stable electrocatalyst for hydrogen generation. Nanoscale 10, 21123-21131 (2018). https://doi.org/10.1039/c8nr07221c

43. J. Palmquist, Z. Czigany, M. Odén, J. Neidhart, L. Hultman, U. Jansson, Magnetron sputtered W-C films with C60 as carbon source. Thin Solid Films 444, 29-37 (2003). https://doi. org/10.1016/s0040-6090(03)00937-4

44. A. Kurlov, A. Gusev, Tungsten carbides and W-C phase diagram. Inorg. Mater. 42, 121-127 (2006). https://doi. org/10.1134/s0020168506020051

45. G. Wang, Z. Gao, G. Wan, S. Lin, P. Yang, Y. Qin, High densities of magnetic nanoparticles supported on graphene fabricated by atomic layer deposition and their use as efficient synergistic microwave absorbers. Nano Res. 7, 704-716 (2014). https://doi.org/10.1007/s 12274-014-0432-0

46. L. Wang, X. Yu, X. Li, J. Zhang, M. Wang, R. Che, Conductive-network enhanced microwave absorption performance from carbon coated defect-rich $\mathrm{Fe}_{2} \mathrm{O}_{3}$ anchored on multiwall carbon nanotubes. Carbon 155, 298-308 (2019). https ://doi.org/10.1016/j.carbon.2019.07.049

47. D. Liu, Y. Du, F. Wang, Y. Wang, L. Cui, H. Zhao, X. Han, MOFs-derived multi-chamber carbon microspheres with enhanced microwave absorption. Carbon 157, 478-485 (2020). https://doi.org/10.1016/j.carbon.2019.10.056

48. F. Wang, M. Wang, X. Han, D. Liu, Y. Wang et al., Coreshell FeCo@carbon nanoparticles encapsulated in polydopamine-derived carbon nanocages for efficient microwave absorption. Carbon 145, 701-711 (2019). https://doi. org/10.1016/j.carbon.2019.01.082

49. A. Ferrari, J. Robertson, Interpretation of Raman spectra of disordered and amorphous carbon. Phys. Rev. B 61, 1409514107 (2000). https://doi.org/10.1103/physrevb.61.14095

50. K. Wang, Y. Wang, Y. Wang, E. Hosono, H. Zhou, Mesoporous carbon nanofibers for supercapacitor application. J. Phys. Chem. C 113, 1093-1097 (2009). https://doi. org/10.1021/jp807463u

51. Y. Du, J. Wang, C. Cui, X. Liu, X. Wang, X. Han, Pure carbon microwave absorbers from anion-exchange resin pyrolysis. Synth. Met. 160, 2191-2196 (2010). https://doi. org/10.1016/j.synthmet.2010.08.008

52. B. Zhao, X. Guo, W. Zhao, J. Deng, B. Fan et al., Facile synthesis of yolk-shell Ni@void@ $\mathrm{SnO}_{2}\left(\mathrm{Ni}_{3} \mathrm{Sn}_{2}\right)$ ternary composites via galvanic replacement/Kirkendall effect and their enhanced microwave absorption properties. Nano Res. 10, 331-343 (2017). https://doi.org/10.1007/s12274-016-1295-3

53. M. Cao, W. Song, Z. Hou, B. Wen, J. Yuan, The effects of temperature and frequency on the dielectric properties, electromagnetic interference shielding and microwave-absorption of short carbon fiber/silica composites. Carbon 48, 788796 (2010). https://doi.org/10.1016/j.carbon.2009.10.028

54. C. Tian, Y. Du, P. Xu, R. Qiang, Y. Wang et al., Constructing uniform core-shell PPy@PANI composites with tunable shell thickness toward enhancement in microwave absorption. ACS Appl. Mater. Interfaces. 7, 20090-20099 (2015). https://doi.org/10.1021/acsami.5b05259 
55. G. He, Y. Duan, H. Pang, Microwave absorption of crystalline Fe/MnO@C nanocapsules embedded in amorphous carbon. Nano-Micro Lett. 12, 57 (2020). https://doi. org/10.1007/s40820-020-0388-4

56. M. Cao, X. Wang, W. Cao, J. Yuan, Ultrathin graphene: electrical properties and highly efficient electromagnetic interference shielding. J. Mater. Chem. C 3, 6589-6599 (2015). https://doi.org/10.1039/c5tc01354b

57. J. Guo, Z. Mao, X. Yan, R. Su, P. Guan et al., Ultrasmall tungsten carbide catalysts stabilized in graphitic layers for high-performance oxygen reduction reaction. Nano Energy 28, 261-268 (2016). https://doi.org/10.1016/j.nanoe n.2016.08.045

58. A. Ohlan, K. Singh, A. Chandra, S. Dhawan, Microwave absorption behavior of core-shell structured poly(3, 4-rthylenedioxy thiophene)-barium ferrite nanocomposites. ACS Appl. Mater. Interfaces. 2, 927-933 (2010). https://doi.org/10.1021/ am900893d

59. Y. Wang, Y. Du, P. Xu, R. Qiang, X. Han, Recent advances in conjugated polymer-based microwave absorbing materials. Polymers 9, 29 (2017). https://doi.org/10.3390/polym9010029

60. Y. Qing, H. Nan, F. Luo, W. Zhou, Nitrogen-doped graphene and titanium carbide nanosheet synergistically reinforced epoxy composites as high-performance microwave absorbers. RSC Adv. 7, 27755-27761 (2017). https://doi.org/10.1039/ c7ra02417g

61. Y. Cheng, M. Tan, P. Hu, X. Zhang, B. Sun et al., Strong and thermostable $\mathrm{SiC}$ nanowires/graphene aerogel with enhanced hydrophobicity and electromagnetic wave absorption property. Appl. Surf. Sci. 448, 138-144 (2018). https:// doi.org/10.1016/j.apsusc.2018.04.132

62. M. Ma, R. Yang, C. Zhang, B. Wang, Z. Zhao et al., Direct large-scale fabrication of $\mathrm{C}$-encapsulated $\mathrm{B}_{4} \mathrm{C}$ nanoparticles with tunable dielectric properties as excellent microwave absorbers. Carbon 148, 504-511 (2019). https://doi. org/10.1016/j.carbon.2019.04.020
63. L. Wang, H. Liu, X. Lv, G. Cui, G. Gu, Facile synthesis 3D porous MXene $\mathrm{Ti}_{3} \mathrm{C}_{2} \mathrm{~T}_{x} @ \mathrm{RGO}$ composite aerogel with excellent dielectric loss and electromagnetic wave absorption. J. Alloys Compd. 828, 154251 (2020). https://doi.org/10.1016/j. jallcom.2020.154251

64. L. Song, Y. Duan, J. Liu, H. Pang, Transformation between nanosheets and nanowires structure in $\mathrm{MnO}_{2}$ upon providing $\mathrm{Co}^{2+}$ ions and applications for microwave absorption. Nano Res. 13, 95-104 (2020). https://doi.org/10.1007/s 1227 4-019-2578-2

65. B. Zhao, G. Shao, B. Fan, W. Zhao, R. Zhang, Investigation of the electromagnetic absorption properties of $\mathrm{Ni} @ \mathrm{TiO}_{2}$ and $\mathrm{Ni} @ \mathrm{SiO}_{2}$ composite microspheres with core-shell structure. Phys. Chem. Chem. Phys. 17, 2531-2539 (2015). https://doi. org/10.1039/c4cp05031b

66. Y. Du, W. Liu, R. Qiang, Y. Wang, X. Han, J. Ma, P. Xu, Shell thickness-dependent microwave absorption of coreshell $\mathrm{Fe}_{3} \mathrm{O}_{4} @ \mathrm{C}$ composites. ACS Appl. Mater. Interfaces. 6, 12997-13006 (2014). https://doi.org/10.1021/am502910d

67. S. Dang, X. Wei, H. Ye, The design theory for a flat microwave absorber with a protective cover. Mater. Res. Express 6, 086312 (2019). https://doi.org/10.1088/2053-1591/ab1df1

68. T. Giannakopoulou, A. Oikonomou, G. Kordas, Double-layer microwave absorbers based on materials with large magnetic and dielectric losses. J. Magn. Magn. Mater. 271, 224-229 (2004). https://doi.org/10.1016/j.jmmm.2003.09.040

69. D. Ding, Y. Wang, X. Li, R. Qiang, P. Xu et al., Rational design of core-shell Co@C microspheres for high-performance microwave absorption. Carbon 111, 722-732 (2017). https://doi.org/10.1016/j.carbon.2016.10.059

70. Z. Ma, C. Cao, Q. Liu, J. Wang, A new method to calculate the degree of electromagnetic impedance matching in one-layer microwave absorbers. Chin. Phys. Lett. 29, 038401 (2012). https://doi.org/10.1088/0256-307x/29/3/038401 